\title{
Electronic Structure and Optical Properties of $\mathrm{Ce}_{1-x} \operatorname{La}_{x}$ PtIn $(0<x<1)$ Compounds by SPR-KKR-CPA and FPLO-CPA Methods
}

\author{
A. JeZIERSKI ${ }^{a, *}$ AND A. SZYTUŁA ${ }^{b}$ \\ ${ }^{a}$ Institute of Molecular Physics, Polish Academy of Sciences, M. Smoluchowskiego 17, 60-179 Poznań, Poland \\ ${ }^{b}$ M. Smoluchowski Institute of Physics, Jagiellonian University, W.S. Reymonta 4, 30-059 Kraków, Poland \\ (Received May 20, 2015; in final form November 23, 2015)

\begin{abstract}
The electronic structure of $\mathrm{Ce}_{1-x} \mathrm{La}_{x} \mathrm{PtIn}$ is studied by means of $a b$ initio full-potential local orbital basis (coherent potential approximation) (FPLO-CPA) and spin polarized relativistic Korringa-Kohn-Rostoker (SPRKKR-CPA) methods within the densities functional methodologies. In both methods, we have observed decrease of the density of states at the Fermi level versus of La concentration. The theoretical photoemission spectra of LaPtIn and CePtIn are compared with the experimental data and the agreement is good. We have also reported the optical properties of LaPtIn and CePtIn compounds obtained in GGA and GGA $+U$ approximation.
\end{abstract}

DOI: 10.12693/APhysPolA.129.109

PACS: 71.15.Ap, 71.15.Mb, 71.20.Eh, 71.23.-k

\section{Introduction}

The physical properties of Ce(La)TIn $(\mathrm{T}=\mathrm{Pt}, \mathrm{Pd})$ compounds were investigated experimentally and theoretically in the last years [1-10]. CePtIn is classified as the Kondo materials with the heat capacity $C_{p} / T$ about $1000 \mathrm{~mJ} \mathrm{~K}{ }^{-2} \mathrm{~mol}^{-1}$ at low temperature [10]. LaPtIn and CePtIn are nonmagnetic with the different values of the densities at the Fermi level [8]. The interesting physical problem is also the change of the electronic structure during the hydrogenation of LaPtIn and CePtIn [9]. The measurements of the electrical resistivity, magnetoresistivity and heat capacity by Ragel et al. [7] indicated that the physical properties of $\mathrm{Ce}_{1-x} \mathrm{La}_{x} \mathrm{PtIn}$ systems changed with the increase of La concentration. The photoemission measurements $[8]$ have shown that the valence band in CePtIn and LaPtIn compounds was formed mainly by Pt $5 d$ and In $5 s$ and $5 p$ states. The experimental results motivated us to study the change of the electronic structure during the substitution Ce by La atoms in $\mathrm{Ce}_{1-x} \mathrm{La}_{x}$ PtIn alloys. We present also the theoretical interpretation of the X-ray photoelectron spectra (XPS) of LaPtIn and CePtIn based on the GGA and GGA $+U$ approximation. To our best knowledge there is no such information in the literature.

The theoretical photoemission spectra and optical properties of LaPtIn and CePtIn are calculated by means of FPLO [11-15] and SPR-KKR [16, 17] methods. The effect of chemical disorder on the electronic structure of $\mathrm{Ce}_{1-x} \mathrm{La}_{x} \mathrm{PtIn}$ alloys is studied by the coherent potential approximation implemented in FPLO-CPA [13] and SPR-KKR-CPA $[16,17]$ methods.

The paper is organized as follows: in Sect. 2 we present the details of the computational methods. The elec-

\footnotetext{
* corresponding author; e-mail:

andrzej · jezierski@ifmpan . poznan.pl
}

tronic and optical properties of LaPtIn and CePtIn as well as the effect of chemical disorder in $\mathrm{Ce}_{1-x} \mathrm{La}_{x} \mathrm{PtIn}$ is discussed in Sect. 3. Finally, we make conclusions and summary.

\section{Computational details}

The band structure of LaPtIn and CePtIn is calculated by using of FPLO-CPA [11-15] and SPR-KKRCPA $[16,17]$ methods. We have performed the selfconsistent band calculations in local density approximation (LDA) within the generalized gradient approximations (GGA) for scalar- and full-relativistic mode. The exchange correlation potential was assumed in the form proposed by Perdew, Burke, and Ernzerhof (PBE) $[18,19]$. The effect of electron correlations was included into GGA $+U$ method $[20,21]$ and the values of $U$ parameters were chosen according to [22]. In the calculations we assumed $U_{\mathrm{Ce}}(4 f)=6.3 \mathrm{eV}, U_{\mathrm{La}}(4 f)=7.5 \mathrm{eV}$ and $U_{\mathrm{Pt}}(4 d)=3.0 \mathrm{eV}$. La(Ce)PtIn compounds crystallize into the hexagonal type structure (space group $P-62 m$, No. 189). The $\mathrm{La}(\mathrm{Ce})$ atoms are located at $3 \mathrm{~g}$ position $(x, 0,1 / 2)$, Pt atoms occupy two positions $1 \mathrm{~b}(0,0,1 / 2)$ and $2 \mathrm{c}(1 / 3,2 / 3,0)$ and In atoms are displayed at $3 \mathrm{f}$ position $(x, 0,0)$. In this work we assumed the value of $x$ position of $\mathrm{La}(\mathrm{Ce})$ and $\mathrm{In}$ as reported in $[5,6]$. The band calculations were performed for the experimental values of lattice parameters: $a=7.823(7.657) \AA$ and $c=4.165$ (4.069) $\AA$ for LaPtIn [1] and CePtIn [1], respectively.

In the calculations of the optical properties we assumed that the electric tensor $\varepsilon_{i j}(\omega)$ consists of an intra and an inter-band part. The intra-band part is calculated by the Drude model, however the inter-band part depended on the band structure. The real part of conductivity $\operatorname{Re} \sigma(\omega)=1 /(4 \pi) \omega \operatorname{Im} \varepsilon(\omega)$ and the energy loss spectrum is given by $L(\omega)=-\operatorname{Im}(1 / \varepsilon(\omega))$.

In this work we present the dependence of inter-band part of the optical conductivity and the loss function on the photon energy. 


\section{Results and discussion}

3.1. The theoretical photoemission spectra of LaPtIn and CePtIn in GGA and $G G A+U$ approximation

The photoemission spectra of LaPtIn and CePtIn have been calculated in GGA and GGA $+U$ approximations using SPR-KKR [16, 17] method. The results are pre-

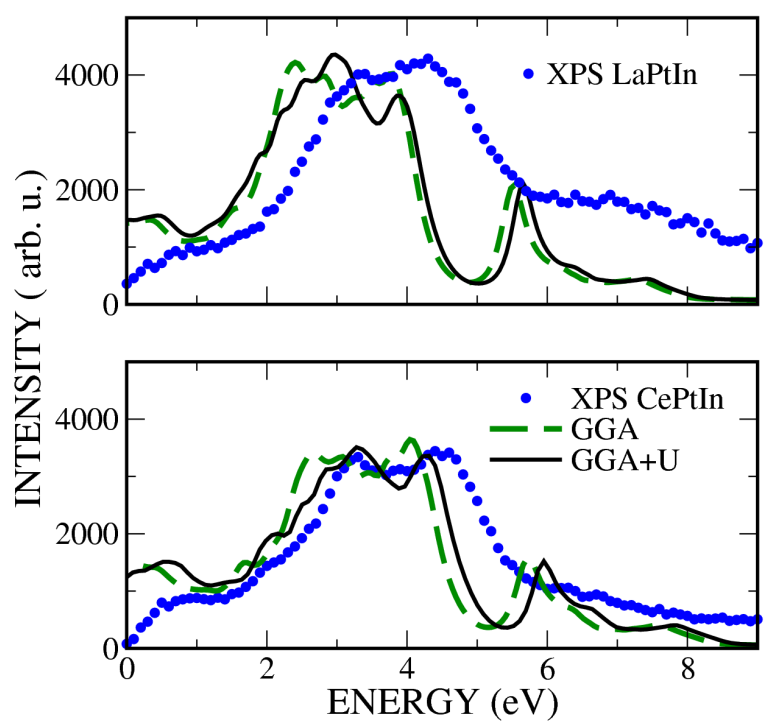

Fig. 1. The theoretical photoemission spectra of LaPtIn (a) and CePtIn (b). The circles denote the experimental data [8]. The solid (broken) curves denote the theoretical results in GGA $(\mathrm{GGA}+U)$ approximation. sented in Fig. 1 for LaPtIn (a) and CePtIn (b), respectively. The experimental values [8] are denoted by the broken curve (XPS). In Fig. 1 the results obtained by GGA and GGA $+U$ are denoted by broken and solid curves, respectively. In the theoretical photoemission spectra we observe the large contribution from $5 d$ states of $\mathrm{Pt}$ and $5 p$ states of In in the region of $2.5<E<4.5 \mathrm{eV}$. The small peak near $E=6 \mathrm{eV}$ is due to $5 s$ states of In. The calculated spectra are close to the experimental results and give the similar contributions from atoms as was suggested from the XPS measurements [8]. The small displacement of the theoretical peaks can be connected with the fact that the calculations were performed for $T=0 \mathrm{~K}$. A good accordance with the experiment data are obtained when we move our theoretical results towards the higher energy about 1.0 and $0.5 \mathrm{eV}$ for LaPtIn and CePtIn, respectively. This result indicates that the values of $U$ parameters [22] which were assumed in the $\mathrm{GGA}+U$ approximation give the similar results as the experimental data, but the positions of peaks are not the same. The change of the values of $U$ parameters did not change significantly the shape and position of the theoretical photoemission spectra.

\subsection{Optical properties of LaPtIn, CePtIn}

In this section, we present the plots of the optical functions: the optical conductivity and the loss function. The optical properties were calculated in GGA and GGA $+U$ approximation. The dependence of the real part of the optical conductivity $\operatorname{Re} \sigma_{x x}(\omega)$ and $\operatorname{Re} \sigma_{z z}(\omega)$ for LaPtIn and CePtIn on the photon energy $\omega$ is visualized in Fig. 2 for GGA (a) and GGA $+U$ (b).
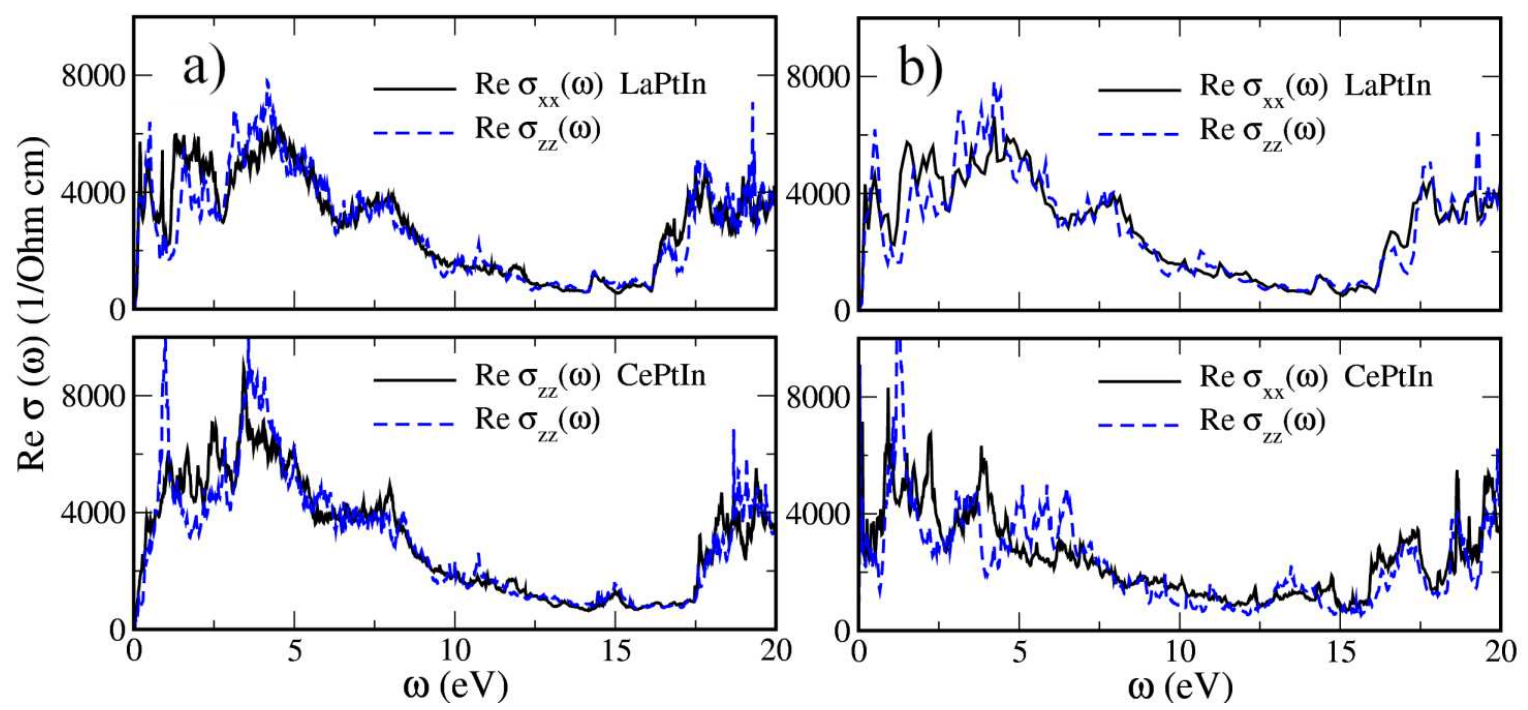

Fig. 2. (a) The dependence of the real part of the optical conductivity Re $\sigma \omega$ ) on the photon energy $\omega$ for LaPtIn and CePtIn in GGA approximation. (b) The same as part (a), but for GGA $+U$ approximation.

The general shape and the positions of the peaks are similar, although the details of the structure are different.
The main peaks are located in the region of $0<\omega<8 \mathrm{eV}$ and above $\omega=17 \mathrm{eV}$. The loss function $L(\omega)$ (Fig. 3) has 
its main peaks in the region of photon energy $12<\omega<$ $17 \mathrm{eV}$, but the structure of this function is different for LaPtIn and CePtIn. The effect of electron correlations is visualized mainly in the spectra of CePtIn (Fig. 3b).
It is difficult to compare our theoretical results to the experimental data, because up to now there is no such information in the literature.
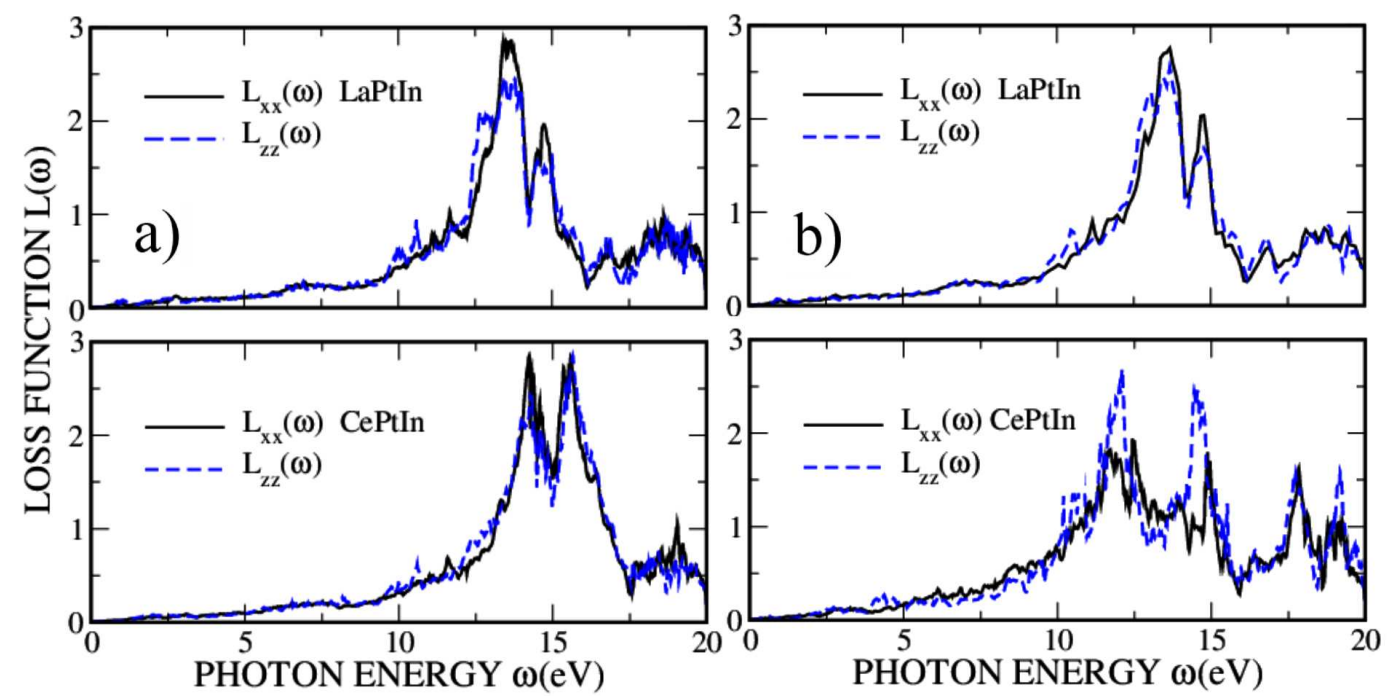

Fig. 3. (a) The plot of the loss function for LaPtIn and CePtIn in GGA approximation. (b) The same as in part (a), but for $\mathrm{GGA}+U$ approximation.

\subsection{Electronic properties of $C e_{1-x} L a_{x}$ PtIn alloys}

In previous sections we have presented the results for LaPtIn and CePtIn systems. It seems that the substitution Ce by La atoms can give new interesting physical results. Experimentally, such systems were studied by Ragel et al. [7]. In order to calculate the change of the densities of states versus concentration we have applied the CPA. We used two different methods: FPLO-CPA and SPR-KKR-CPA. The change of the lattice parameters with the concentration was assumed according to the experimental data [7]. The values of lattice parameters $a$ and $c$ increased linearly with the concentration $x$ (Fig. 1 of [7]).

The electronic densities of states of $\mathrm{Ce}_{1-x} \mathrm{La}_{x} \mathrm{PtIn}$ alloys were calculated for the whole range of concentration $(0<x<1)$ with the step of $x=0.1$. The electronic densities of states obtained by SPR-KKR-CPA method are presented in Fig. 4 for $x=0.2,0.4,0.6$ and 0.8. The electronic density of states (DOS) consists of four main peaks: the peak above the Fermi level located at $3 \mathrm{eV}$ is due to La $4 f$ states. The contribution to the density of states at the Fermi level comes mainly from Ce $4 f$ states. The third broad peak located between $-5.0<E<-2 \mathrm{eV}$ gives the contribution from $\mathrm{Pt}$ $4 d$ states. The last peak near $-6 \mathrm{eV}$ gives the contribution from $4 s$ states of Pt. Results presented in Fig. 4 have shown the strong modification of the electronic densities of states with the change of the concentration $x$.

In Fig. 5 we present the total density of states obtained by two methods: SPR-KKR-CPA and FPLO-

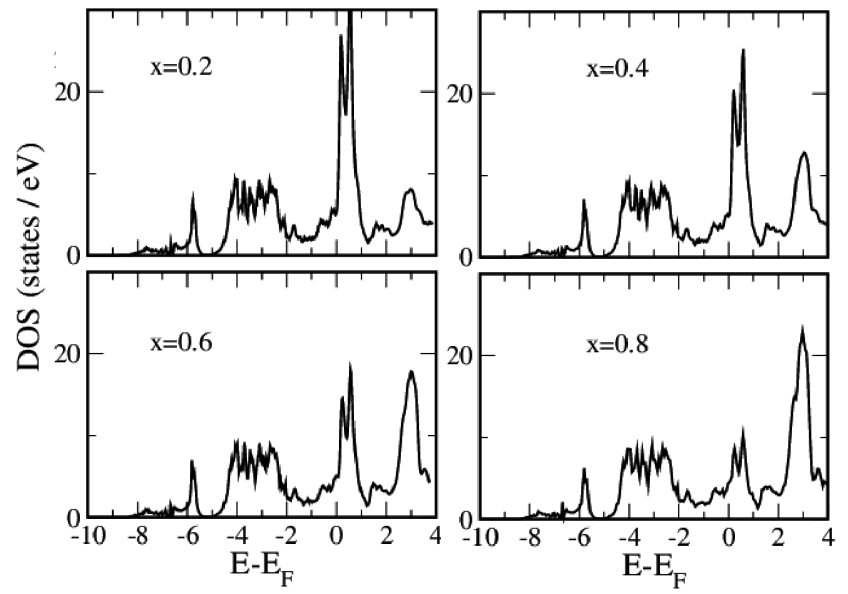

Fig. 4. The total electronic densities of states (DOS) for $\mathrm{Ce}_{1-x} \operatorname{La}_{x} \operatorname{PtIn}(x=0.2,0.4,0.6$ and 0.8$)$.

CPA. The main peaks are located in the similar place, but the structure of peaks is different.

The dependence of the density of states at the Fermi level $N\left(E_{\mathrm{F}}\right)$ on the La concentration obtained by FPLOCPA and SPR-KKR-CPA methods is presented in Fig. 6. This value decreases with increase of $x$. Both methods give the similar dependence of $N\left(E_{\mathrm{F}}\right)$ versus $x$, but the values obtained by FPLO-CPA are lower. The change of the density of states at the Fermi level determines the dependence of the specific heat coefficient. 


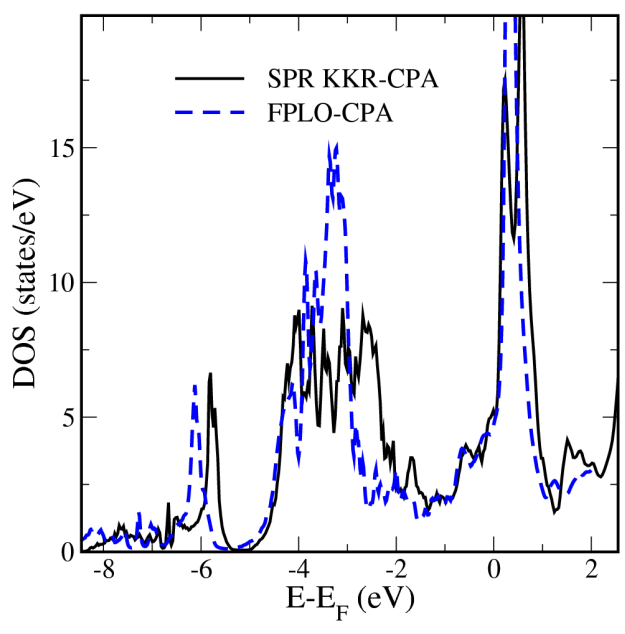

Fig. 5. The total density of states for $\mathrm{Ce}_{0.5} \mathrm{La}_{0.5} \mathrm{PtIn}$. Solid (broken) line denotes the results obtained by SPRKKR-CPA and FPLO-CPA methods, respectively.

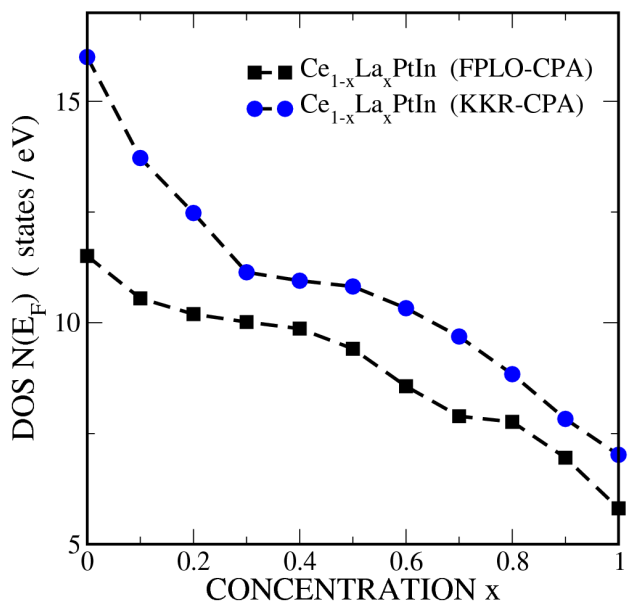

Fig. 6. The dependence of the density of states at the Fermi level $N\left(E_{\mathrm{F}}\right)$ on the concentration in $\mathrm{Ce}_{1-x} \mathrm{La}_{x}$ PtIn. Squares (circles) denote results for FPLO-CPA (SPR-KKR-CPA), respectively.

\section{Conclusions}

LaPtIn and CePtIn systems have many interesting physical properties. In this work we pay our attention on the band structure, optical properties and the influence of chemical disorder in $\mathrm{Ce}_{1-x} \mathrm{La}_{x} \mathrm{PtIn}$. Our $a b$ initio calculations have shown that the results obtained in the GGA $+U$ approximation are close to the XPS data, however the effect of electron correlations is only visualized in intra-band part of the optical functions for CePtIn. The effect of the chemical disorder was studied by using two different $a b$ initio methods: FPLO-CPA and SPRKKR-CPA. In both methods the densities of states decrease with the increase of concentration of La and the densities of states are modified. FPLO-CPA and SPRKKR methods give the similar results, although details are different.

\section{Acknowledgments}

This work (A.J.) was supported by the National Science Centre (NCN) grant number DEC2011/01/B/ST3/02212.

\section{References}

[1] D. Rossi, D. Mazzone, R. Marazza, R. Ferro, Z. Anorg. Allg. Chem. 507, 235 (1983).

[2] A. Szytuła, B. Penc, Ł. Gondek, Acta Phys. Pol. A 111, 475 (2007).

[3] S. Gupta, K.G. Suresh, J. Alloys Comp. 618, 562 (2015).

[4] M. Klicpera, P. Javorsky, Mater. Res. Expr. 1, 06301.1 (2014).

[5] I.J.T. Jensen, H. Fjellvag, B.C. Hauback, J. Alloys Comp. 462, 11 (2008).

[6] W. Hermes, U.Ch. Rodewald, B. Chevalier, R. Pottgen, Solid State Sci. 9, 874 (2007).

[7] F.C. Ragel, P.V. Plessis, A.M. Strydom, J. Phys. Condens. Matter 21, 046008.1 (2009).

[8] A. Jezierski, B. Penc, A. Szytuła, A. Winiarski, Acta Phys. Pol. A 122, 212 (2012).

[9] A. Jezierski, A. Szytuła, J. Solid State Chem. 234, 48 (2016).

[10] K. Satoh, T. Fujita, Y. Maeno, Y. Uwatoko, H. Fujii, J. Phys. Soc. Jpn. 59, 692 (1990).

[11] K. Koepernik, H. Eschrig, Phys. Rev. B 59, 1743 (1999).

[12] I. Opahle, K. Koepernik, H. Eschrig, Phys. Rev. B 60, 14035 (1999).

[13] K. Koepernik, B. Velicky, R. Hayn, H. Eschrig, Phys. Rev. B 55, 5717 (1997).

[14] H. Eschrig, K. Koepernik, I. Chaplygin, J. Solid State Chem. 176, 482 (2003).

[15] H. Eschrig, The Fundamentals of Density Functional Theory, 2nd ed., Edition am Gutenbergplatz, Leipzig 2003.

[16] H. Ebert et al., The Munich SPR-KKR package, version 6.3, http://ebert.cup.unimuenchen.de/SPRKKR.

[17] H. Ebert, D. Kodderitzsch, J. Minar, Rep. Prog. Phys. 74, 096501 (2011).

[18] J.P. Perdew, K. Burke, M. Ernzerhof, Phys. Rev. Lett. 77, 3865 (1996)

[19] J.P. Perdew, K. Burke, M. Ernzerhof, Erratum: Phys. Rev. Lett. 78, 1396 (1997).

[20] S.L. Duradev, C.A. Botton, S.Y. Savrasov, C.J. Humphreys, A.P. Sutton, Phys. Rev. B 57, 1505 (1998).

[21] A.L. Liechtenstein, V.I. Anisimov, J. Zaanen, Phys. Rev. B 52, R 5467 (1995).

[22] S. Curtarolo, W. Setyawan, G.L.W. Hart, M. Jahnatek, R.V. Chepulskii, R.H. Taylor, S. Wang, J. Xue, K. Yang, O. Levy, M.J. Mehl, H.T. Stokes, D.O. Demchenko, D. Morgan, Computat. Mater. Sci. 58, 218 (2012). 